\title{
Fermi Observations of Blazars: Implications for Gamma-ray Production
}

\author{
Juri Poutanen* \\ Astronomy Division, Department of Physics, PO Box 3000, FI-90014 University of Oulu, \\ Finland \\ E-mail: juri.poutanen@oulu.fi
}

\section{Boris E. Stern}

Institute for Nuclear Research, Moscow, Russia

Astro Space Center, Lebedev Physical Institute, Moscow, Russia

E-mail: stern.boris@gmail.com

\begin{abstract}
The brightest blazars detected by the Fermi Gamma-ray Space Telescope Large Area Telescope (Fermi/LAT) show significant breaks in their spectra at a few GeV. The sharpness and the position of the breaks can be well reproduced by absorption of $\gamma$-rays via photon-photon pair production on He II and H I Lyman recombination continua (LyC) produced in the broad-line region (BLR). Using 138 weeks of LAT observations of the brightest GeV blazar 3C 454.3 we find a powerlaw dependence of the peak energy on flux and discover anti-correlation between flux and the column density of the $\mathrm{He}$ II LyC which is responsible for absorption of the $>2.5 \mathrm{GeV}$ photons in this object. The strength and the variability of the absorption implies the location of the $\gamma$-ray emitting zone close to the boundary of the high-ionization part of the BLR and moving away from the black hole when the flux increases. A combination of the $\mathrm{GeV}$ breaks with the detection of a few powerful blazars in the $\mathrm{TeV}$ range puts strong constraints on the BLR size. Additional spectral breaks at $\sim 100$ and $\sim 400 \mathrm{GeV}$ due to absorption by the Balmer and Paschen lines could be detected by the Cherenkov Telescope Array.
\end{abstract}

AGN Physics in the CTA Era - AGN2011,

May 16-17, 2011

Toulouse, France

${ }^{*}$ Speaker. 


\section{Fermi spectra of blazars}

The Fermi/LAT has detected a few hundred blazars in the $100 \mathrm{MeV}-100 \mathrm{GeV}$ range [2]. The high photon statistics from the brightest sources allows also to study their spectra with unprecedented accuracy. These studies have shown that the spectra of high-luminosity sources, flat-spectra radio quasars (FSRQs) and low-energy synchrotron peaked BL Lac objects, are much better described by a broken power law than by a simple powerlaw or any smoothly curved models $[1,3]$ (see Fig. 1a). The works dedicated to 3C $454.3[5,4]$ found the spectral hardness-flux correlation at a nearly constant break energy.

The observed spectral breaks are too sharp to be associated with the cooling or the KleinNishina effects [5]. External Compton scattering on a truncated electron spectrum [1] also does not reproduce the break sharpness. Naïvely, one would think that the break energies, mostly lying below $10 \mathrm{GeV}$ (as measured in the object frame, see Fig. 1a), seem also to be too small to be produced by the $\gamma$-ray absorption due to photon-photon $(\gamma \gamma)$ pair production in the broad-line region (BLR), as the strongest BLR line, $\mathrm{Ly} \alpha$, absorbs $\gamma$-rays starting only from $25.6 \mathrm{GeV}$ [3]. However, the BLR contains not only hydrogen, but also e.g. helium, and emits not only lines, but also strong recombination continua. The observed break energies $[3,18]$ favour the He II and $\mathrm{HI}$ Lyman recombination continua $(\mathrm{LyC})$ as the main opacity source. A relatively high opacity in the He II LyC observed in a number of bright blazars implies the location of the $\gamma$-ray emitting region within the highly ionized inner part of the BLR [18].
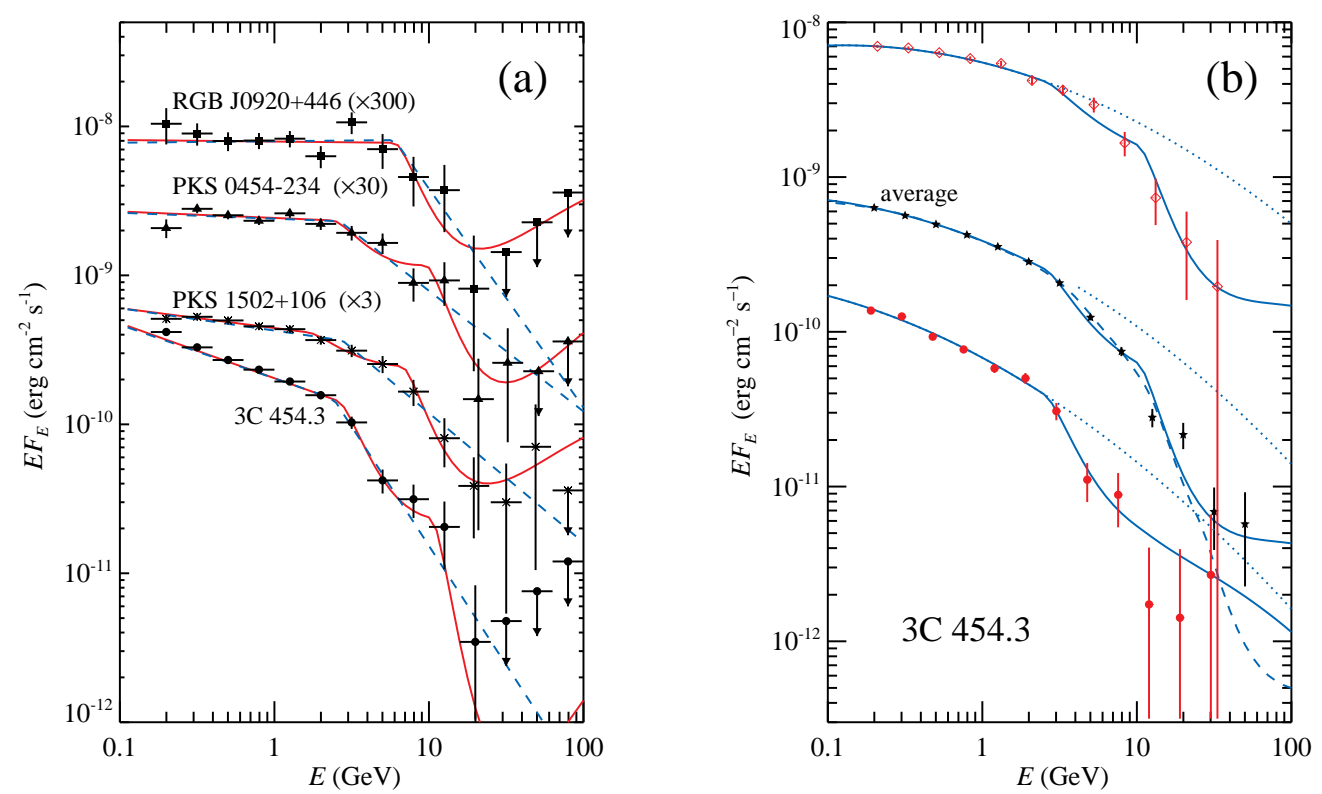

Figure 1: (a) Spectral energy distribution of a few blazars as observed by Fermi/LAT during first 6 months of its operation. The best-fit broken power law and a power law with the absorption by the He II and H LyC (double-absorption) are shown by the dashed and solid lines, respectively. From [18]. (b) Spectral energy distribution of 3C 454.3 at low and high fluxes (intervals B and H, see Fig. 3) as well as averaged over the whole observation period of 2.5 years. Here the underlying continuum is modeled as the lognormal function (shown by the dotted curve). The models modified by double-absorption are shown by solid curves, while the dashed curve is for absorption by BLR at a fixed ionization parameter $\log \xi=2.5$. From [20]. 


\section{Broad-line region and $\mathrm{GeV}$ opacity}

It is well known that BLRs around quasars emit lines associated with different ionization stages, with the broader high-ionization lines being produced closer to the central black hole [16]. Reverberation mapping also demonstrated a strong anti-correlation between the line width and the time delays of the line response to the continuum variations [17]. The BLR size, as measured from the C IV $1549 \AA$ line delays, scales with the accretion luminosity as $R_{\mathrm{C}_{\mathrm{IV}}} \approx 0.2 L_{47}^{1 / 2} \mathrm{pc}$ [12]. However, the delays in the high-ionization He II $1640 \AA$ line as observed in Seyferts are 3-5 times smaller $[13,16]$. On the other hand, the Balmer lines give sizes 2-3 times larger. It is also worth mentioning that the scaling [12] extrapolated to the high-luminosity quasars is based on one object (S5 0836+71) and one line (C IV 1549 $\AA$ ), while the BLR size estimated in the Seyfert-luminosity range has more than an order of magnitude spread. Thus the actual BLR size in quasars is highly uncertain. The strongest BLR line, Ly $\alpha$, does not show any variability on the timescale of years in the four quasars in which it was observed [12], implying that it is produced much further away that C IV 1549 . This has important implications for the opacity of the GeV photons.

Using XSTAR we generated [18] a grid of photoionization models of the BLR clouds assumed to be simple slabs of constant gas density $n_{\mathrm{H}}$, of fixed column density at $N_{\mathrm{H}}=10^{23} \mathrm{~cm}^{-2}$, and a clear view to the ionizing source. We varied the ionization parameter $\xi=L /\left(r^{2} n_{\mathrm{H}}\right)$ from $10^{0.5}$ to $10^{2.5}$. The resulting spectra are shown in Fig. 2a. BLR radiation consists mostly of lines and recombination continua, which in the UV range produce narrow, line-like features, because the temperature of the photoionized regions is typically much below the corresponding ionization potentials of $\mathrm{H}$ and $\mathrm{He}$.

If the $\gamma$-rays are produced within the BLR, they propagate through a roughly isotropic photon field. The absorption strength by the isotropic line photons of energy $E_{0}$ can be characterized by the Thomson optical depth [18]:

$$
\tau_{\mathrm{T}}=N_{\mathrm{ph}} \sigma_{\mathrm{T}} \approx \frac{L_{\text {line }}}{4 \pi R^{2} c E_{0}} R \sigma_{\mathrm{T}} \approx 110 \frac{L_{\text {line }, 45}}{R_{18}} \frac{10 \mathrm{eV}}{E_{0}},
$$

where $N_{\mathrm{ph}}$ is the column density of line photons along the line of sight, $\sigma_{\mathrm{T}}$ is the Thomson crosssection, $L_{\text {line }}$ is the line luminosity, and $R$ is the typical size. (Here we define $Q=10^{x} Q_{x}$ in cgs units.) The energy-dependent opacity is

$$
\tau_{\gamma \gamma}\left(E, E_{0}\right)=\tau_{\mathrm{T}} \frac{\sigma_{\gamma \gamma}(s)}{\sigma_{\mathrm{T}}}
$$

Here $\sigma_{\gamma \gamma}(s)$ is the angle-averaged $\gamma \gamma$ cross-section, which has a threshold at $s \equiv E E_{0} /\left(m_{\mathrm{e}} c^{2}\right)^{2}=1$, i.e.

$$
E_{\mathrm{th}}=261 \mathrm{GeV} /\left(E_{0}[\mathrm{eV}]\right),
$$

rapidly grows to the maximum of $\sim 0.21$ at the energy of $\sim 3.5$ times the threshold, and then slowly decreases roughly inversely with energy $[9,26,6]$. High-energy photons transmitted through the soft line photons are attenuated as $\propto \exp \left(-\tau_{\gamma \gamma}\left(E, E_{0}\right)\right)$. Approximating the spectrum by a power law, we can estimate the break in the power-law index at $E_{\text {th }}$ produced by an individual strong line (or recombination continuum) as

$$
\Delta \Gamma=-\frac{\mathrm{d} \ln \exp \left(-\tau_{\gamma \gamma}\left(E, E_{0}\right)\right)}{\mathrm{d} \ln E} \approx \tau_{\mathrm{T}} \max \frac{\sigma_{\gamma \gamma}(s) / \sigma_{\mathrm{T}}}{\ln s} \approx \frac{\tau_{\mathrm{T}}}{4} .
$$



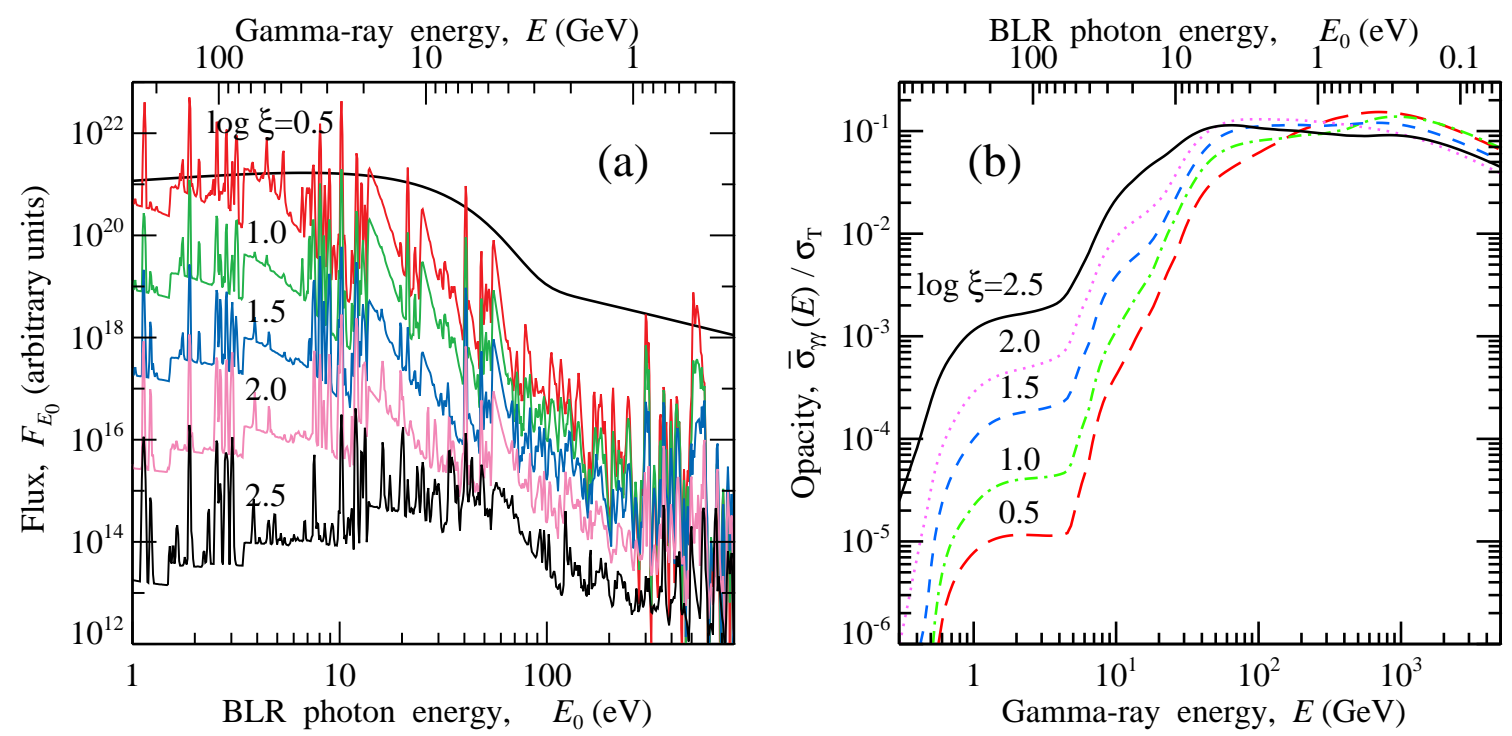

Figure 2: (a) Spectra $F_{E}=d N / d \ln E$ of the BLR clouds of column density $N_{\mathrm{H}}=10^{23} \mathrm{~cm}^{-2}$ and various ionization parameters $\xi$ photoionized by a quasar continuum shown by a smooth curve and consisting of a multicolor disc with the maximum temperature $10^{5} \mathrm{~K}$ and a power law tail of photon index $\Gamma=2$ extending to $100 \mathrm{keV}$ with $10 \%$ of the total luminosity. We use photoionization code XSTAR vs. 2.2 [11]. The upper axis show the threshold energy for $\gamma \gamma$ reaction corresponding to the BLR photon, given by Eq. (2.3). (b) Angle-average cross section (in units of Thomson cross section) for $\gamma$-rays due to $\gamma \gamma$ absorption on the BLR photons with the spectra shown in panel (a). Jumps in opacity are clearly seen at energies corresponding to the strongest lines and recombination continua. The $0.3-0.5 \mathrm{GeV}$ jump for large $\xi$ is produced by $\mathrm{O}$ VIII 16-19 ̊̊ lines, the jump at low $\xi$ is due to the O VII $22 \AA$ line complex. The jump at $\sim 5 \mathrm{GeV}$ visible at all ionizations is due to the $\mathrm{He}$ II $\mathrm{LyC}$ and $\mathrm{Ly}$ lines at $40-60 \mathrm{eV}$. The $20-30 \mathrm{GeV}$ jump seen at low $\xi$ is produced by H I Ly lines and continuum and the C IV 1549 A line. From [18].

Equation (2.1) shows that in powerful quasars with the Ly $\alpha$ luminosity of about $10^{45} \mathrm{erg} \mathrm{s}^{-1}$, the $\gamma$-rays above $30 \mathrm{GeV}$ have troubles of escaping unless the density of the line photons is sufficiently small (e.g. if they are produces in a large volume of $R \gtrsim 3 \mathrm{pc}$ ), or if the $\gamma$-rays are produced outside the BLR. As the BLR size $R$ (in a given line) scales with the total luminosity $L^{1 / 2}$ [12], the opacity also scales similarly (assuming a constant covering factor of BLR clouds)

$$
\tau_{\mathrm{T}} \propto L^{1 / 2}
$$

and obviously the $\gamma \gamma$ absorption can be important only in the high-luminosity sources such as FSRQs. Another important consequence of Eq. (2.1) is that it is not the luminosity, but $L / R$ ratio (i.e. compactness) that determines the role of a specific line in absorption of the $\gamma$-rays.

Because of strong stratification of the BLR extending over two orders of magnitude in distance $[14,15]$, any model assuming that all lines are produced at the same distance from the central source $[10,19,22]$ strongly underestimates the $\gamma \gamma$-opacity by the relatively weak, high-ionization lines produced in a small volume (if the $\gamma$-rays are produced closer to the centre) and overestimates the opacity for multi-GeV photons from the strong, low-ionization lines which are produced in a larger volume. 
The total $\gamma \gamma$-opacity associated with a given BLR shell at a fixed ionization can be determined by a convolution of the opacity for the line radiation with the BLR photon column density spectral distribution

$$
\bar{\tau}_{\gamma \gamma}(E)=\tau_{\mathrm{T}} \frac{\bar{\sigma}_{\gamma \gamma}(E)}{\sigma_{\mathrm{T}}}=\int \sigma_{\gamma \gamma}(s) \frac{d N_{\mathrm{ph}}}{d E_{0}} \mathrm{~d} E_{0} .
$$

The $\gamma \gamma$ cross sections weighted with the BLR spectra from Fig. 2a are shown in Fig. 2b. If ionization is high, the dominant photon source is the He II complex at $40-60 \mathrm{eV}$, which produces a break in opacity at 4-7 GeV. Above $10 \mathrm{GeV}$, the opacity is a rather smooth function of energy with additional absorption coming from $\mathrm{He}$, hydrogen Ly lines and recombination continuum, and $\mathrm{C} \mathrm{V}$ $2274 \AA$. In the $0.3-0.7 \mathrm{GeV}$ region the opacity has another break due to high-ionization lines of $\mathrm{O}$ VII. In the low-ionization environment, the strongest absorption is produced by hydrogen Ly lines and $\mathrm{LyC}$, forcing a break in opacity at about $20-30 \mathrm{GeV}$. At the same time, the break due to helium is also clearly visible at a few $\mathrm{GeV}$. The opacity is nearly flat up to $1 \mathrm{TeV}$ because of the contribution from additional lines: Mg II $2800 \AA, \mathrm{H} \alpha$, and He I $10832 \AA$. As we argued above, the complete model for the opacity has to be necessarily multi-zone and include radial stratification of the BLR and probably the angular dependence of the BLR radiation, which can affect the threshold energies.

\section{Blazars and absorption by He II and H I LyC}

For the first analysis [18], we have selected several brightest FSRQs from the sample of 12 objects in Table 1 of [3] and have chosen the same 180-day interval for easier comparison. We have developed our own software for data analysis and compared its results for the simple broken power law models with those obtained by the standard maximum likelihood analysis tool GTLIKE in [3]. For a few objects the fits with the power-law are acceptable, while other show clear breaks (see Fig. 1a) that are rather well described by the broken power law model (see details in [18]).

To check the hypothesis that the BLR photons are responsible for the breaks, we have applied a simplified model of the BLR consisting of two strongest features: He II LyC at $54.4 \mathrm{eV}$ and $\mathrm{H} \mathrm{I} \mathrm{LyC}$ at $13.6 \mathrm{eV}$. We call this double-absorber (DA) model. The free parameters are the optical depths $\tau_{\mathrm{He}}$ and $\tau_{\mathrm{H}}$ in these "lines". The observed absorption threshold were redshifted by the appropriate $1+z$ factor. Assuming that the underlying continuum is a power-law, we obtained a good fit (see Fig. 1a). In RGB J0920+446 (with $z=2.19$ ), the absorption is seen only at high energies with the break energy corresponding to the pair-production threshold on $\mathrm{H} \mathrm{I} \mathrm{LyC,} 19.2 \mathrm{GeV} /(1+z) \sim 6$ $\mathrm{GeV}$, while in other cases the first break is close to the threshold for the absorption on He II LyC, $4.8 \mathrm{GeV} /(1+z)$, and there are indication of the second break at higher energy due to H I LyC.

The FSRQ 3C 454.3 at redshift $z=0.859$ is by far the brightest blazar in the $\gamma$-ray range during the lifetime of Fermi. The source shows variations in brightness by two orders of magnitude (see Fig. 3). LAT has detected more than hundred thousand photons from this source. With such a rich photon statistics, it is worth trying to study the spectral variation at various flux levels, to constrain the shape of the underlying spectrum, and to search for correlations between flux and the $\gamma \gamma$ absorption depth. The last aim is the most important one, as it allows to learn about variations in the location of the $\gamma$-ray emitting region. 


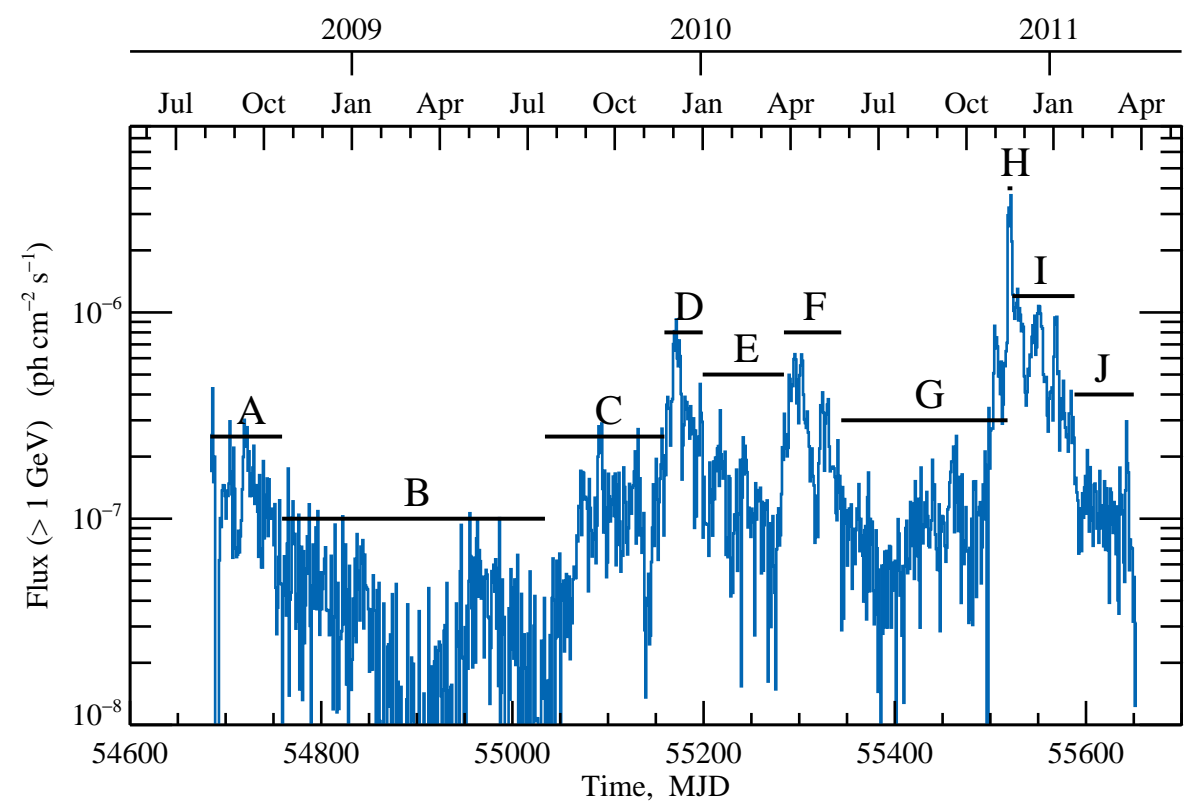

Figure 3: The light curve of FSRQ 3C 454.3 above $1 \mathrm{GeV}$ as observed by Fermi/LAT. Adapted from [20].

We have split the data into the ten time intervals covering periods of more or less constant flux levels and analyzed the spectrum in each interval. The spectra for the high- (interval $\mathrm{H}$ ) and low-flux (B) intervals as well as the average spectrum are shown in Fig. 1b. Clear deviations from a simple power-law spectrum are obvious, and addition of the DA still does not provide a good fit at all flux levels. Thus instead of the power-law to describe the underlying continuum we have tried a lognormal function $E F_{E} \propto 10^{-\log ^{2}\left(E / E_{\mathrm{peak}}\right) / \sigma_{\mathrm{ln}}^{2}}$ plus the DA. The fits with this model are superior compared to the previously used simple phenomenological models, especially at high fluxes with high photon statistics. The data can be also well fitted with $\gamma \gamma$ absorption produced by the full BLR spectrum of high-ionization (see dashed line in Fig. 1b). A large contribution to the $\chi^{2}$ of the spectral fits comes actually from the high-energy tail. This might be an evidence for variations of the absorber optical depth during the observation and the fact that the sum of the spectra with low and high opacities differs from the spectrum at a fixed intermediate opacity. A probable excess of photons above $15 \mathrm{GeV}$ (especially for the average spectrum) can also indicate that the spectrum is a superposition of emission states with different opacities due to the extended or moving emission zone. This proposal is supported by the spectral variability seen during the latest flare in 2010 November [4], where photons above $10 \mathrm{GeV}$ arrive mostly at the end of the flare, clearly indicating the position of the $\gamma$-ray emitting region further away from the black hole.

$3 C 454.3$ demonstrates a highly significant correlation of the peak energy $E_{\text {peak }}$ with flux (similar to the hardness-flux correlation [5]), which is well represented by a power-law (Fig. 4a):

$$
E_{\text {peak }}=(46 \pm 2) \mathrm{MeV} \mathrm{F}_{-9}^{0.60 \pm 0.04}
$$

where $F=E F_{E}$ at $1 \mathrm{GeV}$. The opacity $\tau_{\mathrm{H}} \sim 3-7$ did not vary significantly, but we observed an anti-correlation between $\tau_{\mathrm{He}}$ and the flux (see Fig. $4 \mathrm{~b}$ ):

$$
\tau_{\mathrm{He}}=(2.61 \pm 0.22)-(1.15 \pm 0.47) \log F_{-9} .
$$



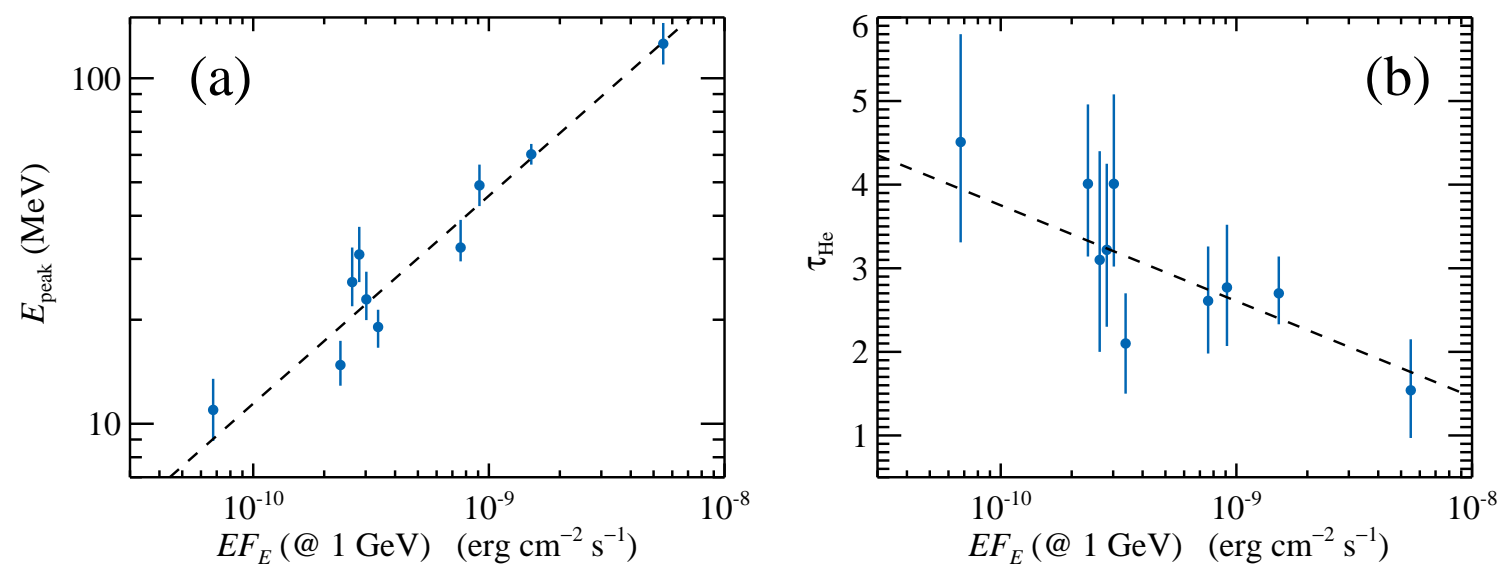

Figure 4: (a) Dependence of the peak (in $E F_{E}$ ) of the best-fitting lognormal (with $\sigma_{\mathrm{ln}}=2.7$ ) + DA model on flux at $1 \mathrm{GeV}$ as observed by Fermi/LAT from 3C 454.3. (b) Dependence of the Thomson optical depth in the He II LyC (defined by Equation (2.1)) on flux at $1 \mathrm{GeV}$. The dashed line shows the best-fitting relation given by Equation (3.2). Adapted from [20].

The strength of absorption at $\mathrm{GeV}$ energies together with the estimations of the luminosities in LyC in principle constraints the BLR size (from Eq. 2.1), where most of this soft radiation is produced. Thus, we get:

$$
R_{\mathrm{He}} \approx 0.22 L_{\mathrm{He}, 44}\left(\tau_{\mathrm{He}} / 3\right) \mathrm{pc}, \quad R_{\mathrm{H}} \approx 3.7 L_{\mathrm{H}, 45}\left(\tau_{\mathrm{H}} / 7\right) \mathrm{pc} .
$$

The luminosities of 3C 454.3 in Ly $\alpha$ and He II $1640 \AA$ are $\sim 10^{45} \mathrm{erg} \mathrm{s}^{-1}$ and $6 \times 10^{43} \mathrm{erg} \mathrm{s}^{-1}$, respectively [25]. Assuming equal luminosities in $\mathrm{LyC}$ and $\mathrm{Ly} \alpha$, the resulting $R_{\mathrm{H}}$ is much larger than the usually quoted sub-pc size of BLR. It is, however, consistent with the absence of Ly $\alpha$ variability in powerful quasars [12]. The He II LyC luminosity is probably larger than $10^{44} \mathrm{erg} \mathrm{s}^{-1}$, but is model dependent and therefore the estimate of $R_{\mathrm{He}}$ is less certain.

A rather large ratio $\tau_{\mathrm{He}} / \tau_{\mathrm{H}}$ (between about $1 / 4$ and 1) and the observed $\tau_{\mathrm{He}}-F$ anti-correlation indicate that the $\gamma$-ray emission region lies close to the boundary $R_{\mathrm{He}}$ of the complete ionization of helium and moves out at higher luminosity. A small distance to the beginning of the $\gamma$-ray emitting region alleviates the problems created by observations of the fast variability from $3 \mathrm{C} 454.3[23,8]$.

\section{Absorption features in the CTA range}

As we have shown in Fig. 2, the strongest spectral features responsible for the $\gamma \gamma$ absorption and the breaks in the Fermi/LAT band are LyC of hydrogen and ionized helium. At energies above $30 \mathrm{GeV}$, there are no significant spectral features expected, just because there are many closelyspaced BLR lines that produce rather smooth absorption profile. However, these simulations only covered a rather narrow set of parameters. The BLR not only has a large spread in the ionization parameter, but the cloud density may also vary by orders of magnitude. For simplicity we fixed $\log \xi$ and looked at the effects of the varying density (see Fig. 5). We see that in the high-density regime, the BLR spectrum below a few eV is dominated by lines of the hydrogen series. Strong Balmer lines $(\mathrm{H} \alpha, \mathrm{H} \beta)$ give a rise to the $\gamma \gamma$ opacity at $100-140 \mathrm{GeV}$ and the $\mathrm{Pa} \alpha$ line is responsible 

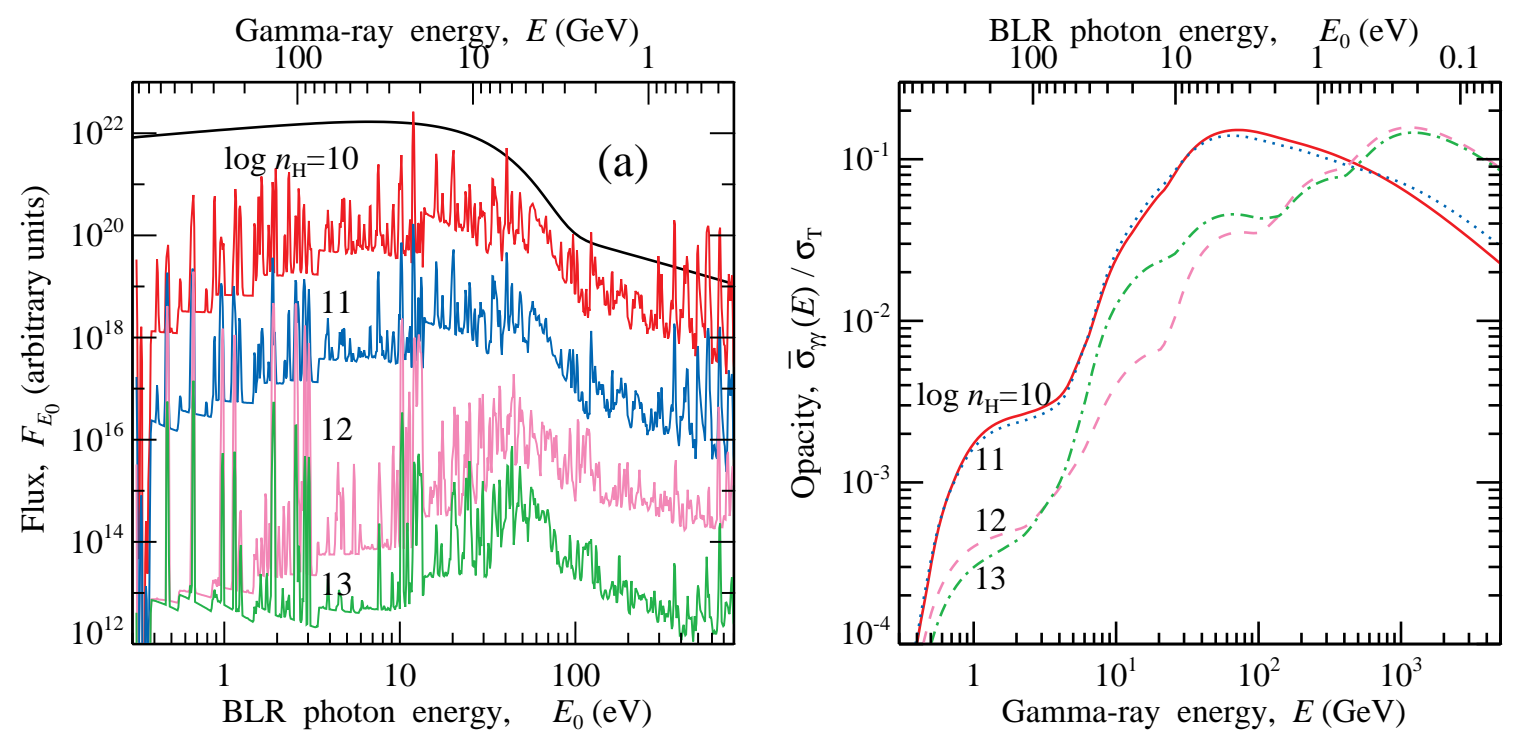

Figure 5: (a) Same as Fig. 2a, but here we fix the ionization parameter at $\log \xi=2.5$ and vary the density of the clouds from $n_{\mathrm{H}}=10^{10}$ (at the top) to $10^{13} \mathrm{~cm}^{-3}$ (at the bottom) with steps of a factor of 10 . (b) Corresponding $\gamma \gamma$ cross sections weighted with the BLR spectrum are shown by the solid, dotted, dashed and dot-dashed curves, respectively. In the high-density regime there are additional jumps in opacity at $\sim 100$ $\mathrm{GeV}$ due to the Balmer lines and at $\sim 400 \mathrm{GeV}$ due to the $\mathrm{Pa} \alpha$ line.

for another rise at $\sim 400 \mathrm{GeV}$. Thus spectral breaks at these energies are expected if multi-GeV photons are transmitted through such a BLR. Detection of such breaks with the Cherenkov Telescope Array (CTA) will give a clue to the structure and physical conditions within the BLR.

\section{Conclusions}

We have shown that the spectral breaks detected by the Fermi/LAT in powerful blazars at a few $\mathrm{GeV}$ can be naturally explained by the $\gamma \gamma$ absorption on the hydrogen and He II LyC. The strength of the absorption ultimately proves that the blazar zone has to be located within or close to the high-ionization zone of the BLR. Variability in the column density of He II LyC that produces absorption above $\sim 3 \mathrm{GeV}$ in the brightest $\mathrm{GeV}$ blazar 3C 454.3 implies that the $\gamma$-ray emitting region is located close to the boundary of fully ionized He zone and is moving away from the black hole when the flux increases. The strength of the $\mathrm{Ly} \alpha$ in this object allows to estimate the BLR size in LyC, which seems to exceed significantly the estimations previously discussed in the literature. A relatively large BLR size dilutes the density of the BLR photons and allows the multi-GeV photons to escape. Detections of a few powerful blazars in the $\mathrm{TeV}$ range thus does not necessarily means that the $\gamma$-ray emitting region is located outside the BLR as argued recently [7, 21, 24]. On the contrary, the $\mathrm{GeV}$ breaks are impossible to produce far away from the BLR as there are no enough photons available for absorption and there is no any other physical mechanism that can produce sharp spectral breaks. High-density clouds embedded within the BLR can produce strong lines of hydrogen series which can absorb multi-GeV photons. Detection of the spectral breaks at $\sim 100$ and $\sim 400 \mathrm{GeV}$ with the CTA will put interesting constraints on the BLR physical conditions and the location of the $\gamma$-ray emission zone. 


\section{Acknowledgments}

This research was supported by the Academy of Finland grant 127512 and the Magnus Ehrnrooth foundation. The research made use of public data obtained from the Fermi Science Support Center. We thank Maxim Barkov, Markus Boettcher, Evgeny Derishev, Chuck Dermer, Gabriele Ghisellini, Julian Krolik, Amir Levinson, Fabricio Tavecchio, and Dmitry Yakovlev for useful discussions and Tim Kallman for his help with XSTAR.

\section{References}

[1] A. A. Abdo et al., Early Fermi Gamma-ray Space Telescope observations of the quasar 3C 454.3, ApJ 699 (2009) 817

[2] A. A. Abdo et al., Bright active galactic nuclei source list from the first three months of the Fermi Large Area Telescope all-sky survey, ApJ 700 (2009) 597

[3] A. A. Abdo et al., Spectral properties of bright Fermi-detected blazars in the gamma-ray band, ApJ 710 (2010) 1271

[4] A. A. Abdo et al., Fermi Gamma-ray Space Telescope observations of the gamma-ray outburst from 3C454.3 in November 2010, ApJ 733 (2011) L26

[5] M. Ackermann et al., Fermi Gamma-ray Space Telescope observations of gamma-ray outbursts from 3C 454.3 in 2009 December and 2010 April, ApJ 721 (2010) 1383

[6] F. A. Aharonian, Very high energy cosmic gamma radiation: a crucial window on the extreme Universe, World Scientific Publishing, River Edge, NJ, 2004

[7] J. Aleksić et al., MAGIC Discovery of Very High Energy Emission from the FSRQ PKS 1222+21, ApJ 730 (2011) L8

[8] G. Bonnoli et al., The $\gamma$-ray brightest days of the blazar 3C 454.3, MNRAS 410 (2011) 368

[9] R. J. Gould \& G. P. Schréder, Pair production in photon-photon collisions, Phys. Rev. 155 (1967) 1404

[10] H. T. Liu \& J. M. Bai, Absorption of 10-200 GeV gamma rays by radiation from broad-line regions in blazars, ApJ 653 (2006) 1089

[11] T. Kallman \& M. Bautista, Photoionization and high-density gas, ApJS 133 (2001) 221

[12] S. Kaspi et al., Reverberation Mapping of High-Luminosity Quasars: First Results, ApJ 659 (2007) 997

[13] K. T. Korista et al., Steps toward determination of the size and structure of the broad-line region in active galatic nuclei. 8: an intensive HST, IUE, and ground-based study of NGC 5548, ApJS 97 (1995) 285

[14] J. H. Krolik, Active galactic nuclei: from the central black hole to the galactic environment, Princeton University Press, Princeton, NJ, 1999

[15] D. E. Osterbrock, G. J. Ferland, Astrophysics of gaseous nebulae and active galactic nuclei, University Science Books, Sausalito, CA, 2006

[16] B. M. Peterson \& A. Wandel, Keplerian Motion of Broad-Line Region Gas as Evidence for Supermassive Black Holes in Active Galactic Nuclei, ApJ 521 (2000) L95 
[17] B. M. Peterson \& A. Wandel, Evidence for Supermassive Black Holes in Active Galactic Nuclei from Emission-Line Reverberation, ApJ 540 (2000) L13

[18] J. Poutanen \& B. E. Stern, GeV breaks in blazars as a result of gamma-ray absorption within the broad-line region, ApJ 717 (2010) L118

[19] A. Reimer, The redshift dependence of gamma-ray absorption in the environments of strong-line AGNs, ApJ 665 (2007) 1023

[20] B. E. Stern \& J. Poutanen, Variation of the $\gamma \gamma$ opacity by the He II Lyman continuum constrains the location of the $\gamma$-ray emission region in the blazar 3C 454.3, MNRAS, in press (2011) [arXiv:1105.2762]

[21] Y. T. Tanaka et al., Fermi Large Area Telescope Detection of Bright $\gamma$-Ray Outbursts from the Peculiar Quasar 4C +21.35, ApJ 733 (2011) 19

[22] F. Tavecchio \& D. Mazin, Intrinsic absorption in 3C 279 at GeV-TeV energies and consequences for estimates of the extragalactic background light, MNRAS 392 (2009) L40

[23] F. Tavecchio, G. Ghisellini, G. Bonnoli, G. Ghirlanda, Constraining the location of the emitting region in Fermi blazars through rapid $\gamma$-ray variability, MNRAS 405 (2010) L94

[24] F. Tavecchio et al., On the origin of the gamma-ray emission from the flaring blazar PKS 1222+216, $A \& A$, in press (2011) [arXiv:1104.0048]

[25] B. J. Wills Hubble Space Telescope Sample of Radio-loud Quasars: Ultraviolet Spectra of the First 31 Quasars, ApJ 447 (1995) 139

[26] A. A. Zdziarski, Saturated pair-photon cascades on isotropic background photons, ApJ 335 (1988) 786 